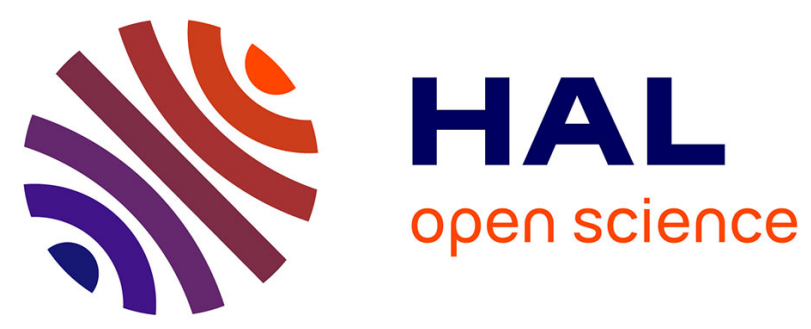

\title{
Jouer son blues quand la dépression résiste... : évaluation de l'efficacité d'un programme de musicothérapie pour des personnes présentant un trouble dépressif et/ou bipolaire résistants
}

Laurie d'Abbadie de Nodrest, Jean-Luc Sudres, Laurent Schmitt, Antoine Yrondi

\section{To cite this version:}

Laurie d'Abbadie de Nodrest, Jean-Luc Sudres, Laurent Schmitt, Antoine Yrondi. Jouer son blues quand la dépression résiste... : évaluation de l'efficacité d'un programme de musicothérapie pour des personnes présentant un trouble dépressif et/ou bipolaire résistants. Annales Médico-Psychologiques, Revue Psychiatrique, 2018, 176 (3), pp.266-272. 10.1016/j.amp.2016.10.013 . hal-01830568

\section{HAL Id: hal-01830568 \\ https://hal-univ-tlse2.archives-ouvertes.fr/hal-01830568}

Submitted on 5 Jul 2018

HAL is a multi-disciplinary open access archive for the deposit and dissemination of scientific research documents, whether they are published or not. The documents may come from teaching and research institutions in France or abroad, or from public or private research centers.
L'archive ouverte pluridisciplinaire HAL, est destinée au dépôt et à la diffusion de documents scientifiques de niveau recherche, publiés ou non, émanant des établissements d'enseignement et de recherche français ou étrangers, des laboratoires publics ou privés. 
Jouer son blues quand la dépression résiste... :

Evaluation de l'efficacité d'un programme de Musicothérapie pour des personnes présentant un trouble dépressif et/ou bipolaire résistants

D’Abbadie de Nodrest, L., Sudres, J-L., Schmitt, L., \& Yrondi, A.

Play its blues when depression withstands...

Assessing effectiveness of a Music-therapy program in a population suffering from resistant depressive disorder and/or bipolar disorder

D’Abbadie de Nodrest, L.*, Sudres, J-L.**, Schmitt, L.***, \& Yrondi, A.****

*D'Abbadie de Nodrest, L. :

Centres d'Etudes et de Recherches en Psychopathologie et Psychologie de la Santé (CERPPS), Université Toulouse II - Jean Jaurès. UFR de Psychologie, 5 allées Antonio Machado. F- 31058 Toulouse Cedex 9.

Portable: 06-83-30-14-96. Mail : lauriedabbadie@gmail.com 


\section{Résumé}

Objectif. - Il n'existe aucune recherche étudiant simultanément les troubles dépressifs et/ou bipolaires résistants et l'impact de la Musicothérapie (réceptive, active, créative) sur ces derniers. Les objectifs de cette étude exploratoire sont d'évaluer la capacité à être dans l'instant présent, les mécanismes de défense, la sévérité de la dépression, le niveau alexithymique, la distorsion corporelle, avant, après et bien après la participation à des ateliers de Musicothérapie. Le vécu des ateliers est également pris en compte pendant les séances et après l'ensemble de celles-ci. Participants et méthodes. - Quarante hommes et femmes ont assisté aux ateliers, quinze ont pu être pris en compte. Hospitalisées dans le «Centre Expert Dépression résistante » du CHU de Purpan, ces personnes ont participé au programme Musicothérapeutique, créé un $\mathrm{CD}$, complété le Five Facets Mindfulness Questionnaire (FFMQ), le Defense Style Questionnaire (DSQ-40), le Beck Depression Inventory (BDI-II), le Bermond-Vorst Alexithymia Questionnaire (BVAQ), le Body Distortion Questionnaire (BDQ), et enfin une échelle créée pour les besoins de l'étude mesurant le vécu par séance, ainsi que l'Echelle Clinique des Thérapies Médiatisées. Résultats. L'analyse statistique suggère des différences significatives entre le t0 (pré-test) et le t1 (post-test) en lien avec les données qualitatives. Discussion et conclusion. - Des améliorations sont repérées à tous les niveaux observés (pleine conscience, défenses, degré de dépression, émotions, distorsion corporelle). L'impact positif des ateliers reste néanmoins à confirmer lors d'une étude longitudinale.

Mots clefs : dépression résistante; Musicothérapie ; émotions ; corps ; mécanismes de défense 


\begin{abstract}
Objective. - There is no research studying resistant depression and/ or resistant bipolar disorders and the impact of Music-Therapy (receptive, active, creative) on those troubles. The aims of this exploratory study were to assess the mindfulness, defense mechanisms, depressive state, alexithymia, body distortion, before, after, and long after the participation in Music-Therapy workshops. The patient's experience is also taken into account during these workshops and after all the sessions. Participants and methods. - Forty men and women participated in workshops but only fifteen were retained. Hospitalized in the " "Treatment-Resistant Depression Expert Center" » based in the Purpan hospital, they participated in the Music-Therapy program, created a CD, completed the Five Facets Mindfulness Questionnaire (FFMQ), the Defense Style Questionnaire (DSQ-40), the Beck Depression Inventory (BDI-II), the Bermond-Vorst Alexithymia Questionnaire (BVAQ), the Body Distortion Questionnaire (BDQ), along with a scale created for the study measuring the feeling at each meeting, and the Clinical Scale of Mediatized Therapies. Results. Statistical analyses showed more than fifteen significant differencies between t0 (pretest) and t1 (post-test) in connection with qualitative results. Discussion and conclusion. - Improvements have been found in all the levels observed (Mindfulness, defenses, depression, emotions, body distortion). The positive impact of Music-Therapy should be confirmed by a longitudinal study.
\end{abstract}

Mots clefs: resistant depression; Music-therapy ; emotions ; body ; defense mechanisms 


\section{Résumé}

Objectif. - Il n'existe aucune recherche étudiant simultanément les troubles dépressifs et/ou bipolaires résistants et l'impact de la Musicothérapie (réceptive, active, créative) sur ces derniers. Les objectifs de cette étude exploratoire sont d'évaluer la capacité à être dans l'instant présent, les mécanismes de défense, la sévérité de la dépression, le niveau alexithymique, la distorsion corporelle, avant, après et bien après la participation à des ateliers de Musicothérapie. Le vécu des ateliers est également pris en compte pendant les séances et après l'ensemble de celles-ci. Participants et méthodes. - Quarante hommes et femmes ont assisté aux ateliers, quinze ont pu être pris en compte. Hospitalisées dans le «Centre Expert Dépression résistante » du CHU de Purpan, ces personnes ont participé au programme Musicothérapeutique, créé un $\mathrm{CD}$, complété le Five Facets Mindfulness Questionnaire (FFMQ), le Defense Style Questionnaire (DSQ-40), le Beck Depression Inventory (BDI-II), le Bermond-Vorst Alexithymia Questionnaire (BVAQ), le Body Distortion Questionnaire (BDQ), et enfin une échelle créée pour les besoins de l'étude mesurant le vécu par séance, ainsi que l'Echelle Clinique des Thérapies Médiatisées. Résultats. L'analyse statistique suggère des différences significatives entre le t0 (pré-test) et le t1 (post-test) en lien avec les données qualitatives. Discussion et conclusion. - Des améliorations sont repérées à tous les niveaux observés (pleine conscience, défenses, degré de dépression, émotions, distorsion corporelle). L'impact positif des ateliers reste néanmoins à confirmer lors d'une étude longitudinale.

Mots clefs : dépression résistante; Musicothérapie ; émotions ; corps ; mécanismes de défense 


\begin{abstract}
Objective. - There is no research studying resistant depression and/ or resistant bipolar disorders and the impact of Music-Therapy (receptive, active, creative) on those troubles. The aims of this exploratory study were to assess the mindfulness, defense mechanisms, depressive state, alexithymia, body distortion, before, after, and long after the participation in MusicTherapy workshops. The patient's experience is also taken into account during these workshops and after all the sessions. Participants and methods. - Forty men and women participated in workshops but only fifteen were retained. Hospitalized in the «"Treatment-Resistant Depression Expert Center" » based in the Purpan hospital, they participated in the Music-Therapy program, created a CD, completed the Five Facets Mindfulness Questionnaire (FFMQ), the Defense Style Questionnaire (DSQ-40), the Beck Depression Inventory (BDI-II), the Bermond-Vorst Alexithymia Questionnaire (BVAQ), the Body Distortion Questionnaire (BDQ), along with a scale created for the study measuring the feeling at each meeting, and the Clinical Scale of Mediatized Therapies. Results. Statistical analyses showed more than fifteen significant differencies between $\mathrm{t} 0$ (pre-test) and $\mathrm{t} 1$ (post-test) in connection with qualitative results. Discussion and conclusion. - Improvements have been found in all the levels observed (Mindfulness, defenses, depression, emotions, body distortion). The positive impact of Music-Therapy should be confirmed by a longitudinal study.
\end{abstract}

Mots clefs: resistant depression; Music-therapy; emotions; body; defense mechanisms 
Qu'elle soit bipolaire ou unipolaire, la dépression résistante fait l'objet de plus d'une dizaine de définitions différentes [3]. En guise de consensus, elle est considérée comme résistante lorsqu'il n'y a pas de réponse à au moins deux antidépresseurs de différentes classes [28]. Parmi les 350 millions de personnes qui seraient atteintes par la dépression (Organisation Mondiale de la Santé, 2015), jusqu'à 15\% de celles-ci en présentent éventuellement une résistante au traitement [3]. De plus, des traces biologiques et psychologiques persistantes de la « dépression-maladie » sont retrouvées. En effet, nous ne sortons pas indemnes de dépressions voire d'hospitalisations répétées. Les patients auraient notamment accès plus difficilement à leurs souvenirs positifs. Nombre d'entre eux acquièrent une vision négative d'eux-mêmes, du monde actuel qui leur en demande trop, de l'avenir. La répétition de plusieurs dépressions les conforte dans ces croyances que la réalité vient confirmer. Lors de la «guérison », ces pensées négatives disparaissent de la conscience, mais perdurent, susceptibles d'être réactualisées par de nouveaux traumatismes ou des modifications durables de l'humeur. De dépression en dépression, elles se retrouveraient moins enfouies, prêtes à réapparaître à l'occasion de traumatismes de plus en plus légers. La vulnérabilité est donc extrême et les soigner est devenu difficile [17]. Une forte pression économique et sociale serait liée à cette difficulté, qui est associée à une augmentation de 40 à 50\% des coûts des soins médicaux directs et indirects, par rapport à la dépression non-résistante [28].

Si nous abordons les diverses tentatives de distinction entre dépression «bipolaire » et «unipolaire », il importe d'admettre qu'il n'existe pas de traits pathognomoniques pour autant. Nonobstant, les données scientifiques suggèrent que certaines caractéristiques évolutives, ou en termes de réponse au traitement, ou encore d'histoire familiale seraient de bons indicateurs afin d'améliorer notre discernement. Ainsi, parmi les signes survenant plus spécifiquement dans les 
dépressions bipolaires, les auteurs relèvent : un début aigu de l'épisode, des caractéristiques atypiques (hyperphagie/ prise de poids, hypersomnie), un ralentissement psychomoteur, des symptômes psychotiques, des caractéristiques mélancoliques, des idées de culpabilité et de mésestime de soi, des idées suicidaires, un émoussement affectif, ainsi qu'une réduction de la concentration. A l'inverse, la présence d'une anorexie ou d'une perte de poids, d'une insomnie, d'un niveau d'activité maintenu, de tristesse, de plaintes cognitives et somatiques, orienteraient d'avantage le diagnostic vers une dépression unipolaire. Les épisodes dépressifs bipolaires seraient plus brefs, d'avantage récurrents, et nous assisterions aussi à une saisonnalité des dépressions. Le premier épisode dépressif apparaitrait plus tôt dans le cas d'une bipolarité et l'effet des antidépresseurs aurait tendance à plus souvent s'épuiser. En outre, la dépression mixte (associée à minimum 3 symptômes d'hypomanie en une semaine) serait 2 fois plus fréquente dans les dépressions bipolaires. Il en irait de même concernant la présence de comorbidités psychiatriques et somatiques. Un autre signe se rapporte aux antécédents familiaux, représentant un argument primordial dans l'identification d'un risque de bipolarité. Enfin, dans les types I et II du trouble bipolaire, nous retrouvons un taux plus élevé de phobie sociale, d'abus de substance, de TOC, de tentatives suicidaires et de THADA que dans la dépression unipolaire [26].

\section{Le lien Musicothérapie-Dépression : une rencontre antérieure à l'Antiquité}

Les plus anciennes histoires relatives à ce lien concernent les figures bibliques de Saül (souvent considéré comme possédé, maniaco-dépressif ou «mélancolique ») et David. Présentant des troubles mentaux après que Yahvé ait envoyé un mauvais esprit, Saül trouvera de l'apaisement grâce David qui lui jouera de la musique [6]. De plus, bien que les effets musicaux 
aient été reconnus bien avant l'Antiquité, la dépression et les troubles bipolaires furent évoqués (sous des termes différents) durant cette période [26], tout comme les vertus bénéfiques de la musique afin de pallier à certains symptômes [16, 22]. Dans le domaine de la Psychiatrie française, Pinel en 1801 relève la présence d'alternances manie/ mélancolie et Esquirol (1838) introduit le terme d'«accès » pour désigner ces états, suggérant leur aspect transitoire et potentiellement récurrent [26]. Esquirol fera aussi partie de ceux qui introduiront la Musicothérapie en Psychiatrie à l'hôpital de la Salpêtrière entre 1820 et 1880 [22].

Seuls quelques travaux démontrent l'impact de la Musicothérapie en réponse à la symptomatologie dépressive. En effet, auprès de sujets souffrant de douleur chronique bégnine, la Musicothérapie réceptive améliore l'«empowerment» (niveau de choix, de décision, d'influence et de contrôle que les usagers des services de santé peuvent exercer sur les évènements de leur vie. cf. OMS, 2015), diminue la douleur chronique, l'état dépressif et l'invalidité (due à la douleur chronique) [29]. De plus, Eckhardt et Dinsmore (2012) décrivent un protocole de «Mindful Music Listenning », liant Musicothérapie réceptive et thérapie de pleine conscience. Dans leur article, ils notent que la Musicothérapie réceptive renforce l'alliance thérapeutique, provoque des améliorations sur le plan physique, émotionnel, communicationnel ou encore de la symptomatologie dépressive (fatigue, faible estime de soi, détresse émotionnelle) [14]. L'article de Lee et Thyer (2013) relève chez le groupe de dépressifs majeurs ayant bénéficié (en plus d'une psychothérapie habituelle) de séances musicothérapeutiques, une atténuation significative des symptômes dépressifs ainsi que de l'anxiété [25]. Le même constat sera réalisé trois mois plus tard. En outre, l'étude de Petchkovsky et al. (2013) souligne une amélioration significative des symptômes dépressifs pour le groupe participant à l'atelier chorale [27]. Ainsi, au vu de la littérature, écouter ou jouer de la musique dans un cadre spécifique a de multiples 
conséquences positives sur la symptomatologie dépressive. Or, certaines limites en lien avec le design de recherche empêchent la reproductibilité des expériences décrites. En effet, les auteurs ont généralement tendance à uniquement mesurer un état dépressif global. Les études investiguées sur le long terme sont peu nombreuses. Peu de techniques actives ou créatives sont proposées dans un cadre structuré. En ajout à cela, rares sont les travaux avec groupes contrôles se rapportant à cette forme d'approche thérapeutique médiatisée. Enfin, le traitement pris par les sujets, le diagnostic posé ou le type de Musicothérapie utilisé sont relativement peu décrits.

\section{Mise en place des objectifs de recherche}

Une étude exploratoire a donc été imaginée, dont l'objectif est de découvrir les effets d'un dispositif de Musicothérapie auprès de personnes présentant un trouble dépressif et/ ou bipolaire cliniquement résistant à deux chimiothérapies. L'hypothèse générale étant que les séances de Musicothérapie ont un impact sur la symptomatologie dépressive et/ ou bipolaire résistante.

Le dispositif est composé d'ateliers comprenant chacun trois types de Musicothérapie : la Musicothérapie réceptive (audition de sons dans un cadre relationnel spécifique, avec des techniques adaptées aux objectifs fixés) [22]; la Musicothérapie active (expression par l'intermédiaire d'objets sonores, d'instruments, ou avec le corps et/ ou la voix.) [22] ; et la Musicothérapie créative (approche créée par Sorabella en 2014, créateur d'un test projectif sonore). Parmi les difficultés retrouvées dans la symptomatologie dépressive bipolaire et/ ou unipolaire, celles retenues pour élaborer un programme structuré sont: le ralentissement psychomoteur [8, 24], la distorsion corporelle [7, 25], la prosodie monocorde [17, 24], la modification rythmique du discours (DSM-V), l'alexithymie; [1,9,15,31], les aspects défensifs $[1,10,11,13]$, ou encore l'incapacité à être dans l'instant présent $[21,24]$. D'autre éléments ont été 
travaillés comme la norme [8,16,23], le repli social [8], l'indécision [19,24] et la créativité $[2,20]$.

\section{Dispositif, participants et méthodes}

\section{Dispositif}

Au vu de la littérature et de la clinique psychopathologique, un programme structuré de Musicothérapie a été élaboré en réponse aux difficultés citées supra pouvant être présentes dans la symptomatologie dépressive résistante. Ce programme comprenait six ateliers de Musicothérapie (réceptive, active, créative) renouvelables, axés sur des objectifs constants qui proposaient de : stimuler la voix, consolider l'adaptation sociale, encourager l'expression émotionnelle, mettre en mouvement le corps (soutien sonore, rythmique), développer la créativité, la capacité à focaliser son attention dans l'instant présent, l'écoute, la communication, la confiance en soi et en l'autre. Chaque atelier proposait une certaine rythmicité avec une alternance entre temps fort (Musicothérapie active, créative) et temps faible, de réparation, en termes de Musicothérapie réceptive, inspirée de la technique du «Mindful Music Listening ». Enfin, des tâches interséances étaient à réaliser trois jours après l'atelier précédent. Le fil rouge de ces travaux se basait sur l'encouragement à la fantasmatisation, l'imagination de sons, de mots d'images à partir d'un thème-support, choisi en amont par le groupe.

\section{Participants et procédure}

Dans cette recherche, les personnes rencontrées sont hospitalisées ou en ambulatoire dans le «Centre Expert Dépression Résistante » du service de Psychiatrie du CHU de Purpan. Après avoir donné leur consentement libre et éclairé, 40 personnes ont participé aux ateliers. Seulement 
15 sujets ont été présents à minimum 4 séances et ont rempli les questionnaires avant, pendant, puis après l'ensemble des ateliers de Musicothérapie. Seules les données complètes et les participants présentant un diagnostic de trouble dépressif unipolaire ou bipolaire résistant ont été retenus dans l'échantillon de départ. Ainsi, en écho avec le fonctionnement réel de la prise en charge, la population de l'étude est de 15 adultes. L'âge de ces derniers est compris entre 26 et 79 ans $(M=54.13 ; E T=17.67)$. L'échantillon se compose de $60 \%$ de femmes et de $40 \%$ d'hommes. Cette recherche leur fut présentée oralement et par écrit comme une étude visant à mieux comprendre le ressenti quotidien de chacun et à améliorer la prise en charge. Une page du questionnaire a permis de renseigner certaines données socio-biographiques comme l'âge, le sexe, les antécédents psychiatriques, le rapport passé et actuel à la musique. Les informations anamnestiques obtenues sont présentées dans le Tableau 1. Enfin, les outils méthodologiques suivants étaient des échelles d'auto-évaluation.

\section{Mesures}

$L^{\prime}$ « ici et maintenant ». Les participants ont complété à deux reprises (avant et après l'ensemble des ateliers) le Five Facets Mindfulness Questionnaire (FFMQ). Cet autoquestionnaire évalue la capacité à focaliser sont état de conscience dans l'instant présent. Initialement élaborée par Baer et al. (2006), a été validée en langue française par Heeren, Douilliez, Peschard, Debrauwere, et Philippot (2011). Cet outil comprend 39 items regroupés en 5 catégories : Observation, Description de l'expérience vécue, Action/ agir en pleine conscience, Acceptation/ non réactivité aux évènements et Absence de jugement. Les sujets doivent indiquer sur une échelle de Lickert en 5 points, allant de 1 («Jamais ou Très rarement vraie ») à 5 («Très 
souvent ou toujours vraie ») dans quelle(s) mesure(s) l'affirmation présentée (exemple: «je perçois mes émotions et sentiments sans devoir y réagir ») est généralement vraie pour eux au cours des quatre derniers jours. Le score total est obtenu par addition des résultats respectifs des 5 facteurs. Plus ce score est important, plus la personne est apte à être en état de pleine conscience. L'alpha de Cronbach pour cette échelle était supérieur à .75 ( $\alpha=.73$ pour notre recherche) [18]

Les mécanismes de défense. Le Defense Style Questionnaire à 40 items (DSQ-40) a été créé par Andrews et al. (1993), traduit en français par Chabrol et Sutter (1996) et validé dans une population française par Bonsack et al. (1998). Cet auto-questionnaire propose l'évaluation du fonctionnement défensif de la personne. Cet outil, présenté à deux reprises $(\mathrm{t} 0 ; \mathrm{t} 1)$, distingue trois styles défensifs : mature (sublimation, humour, anticipation, répression), névrotique (annulation, pseudo-altruisme, idéalisation, formation réactionnelle) et immature (projection, agression passive, acting out, isolation, omnipotence-dévaluation, rêverie autistique, déni, déplacement, dissociation, clivage, rationalisation, somatisation) [12]. Pour chaque item, le participant répond sur une échelle de Lickert («accord/ désaccord») en 9 points (médiane : 3). Le score d'un sujet pour un mécanisme de défense est la moyenne des scores des items explorant un mécanisme [12]. Par rapport aux autres facteurs, le premier (style immature) a une consistance interne suffisante avec un alpha de Chronbach de .71.

La symptomatologie dépressive. Les personnes ont complété en t0 et t1 le Beck Depression Inventory (BDI), créé à l'origine par Beck en 1961, puis révisé sous le nom de BDI-1A puis BDI-II (1996). Cet auto-questionnaire comprend 21 items concernant un ensemble de symptômes dépressifs courants. Chaque item est coté de 0 à 3 en fonction de la sévérité de l'état relatif au thème. Le score total peut s'étendre de 0 à 63 . Plus il est élevé, plus la sévérité des symptômes dépressifs est importante. D’après, Beck, Steer et Brown (1998), le BDI-II permet de mettre en 
évidence un degré de dépression et non de poser un diagnostic. Il fournit une note totale, une estimation d'intensité globale de la dépression. Ainsi, dans leur version française, une moyenne de 22.69 (ET : 10.92) correspond à une dépression légère, les participants ayant un score total moyen de 29.91 (ET : 11.34) auraient une dépression moyenne. Enfin, nous parlons de degré sévère de dépression lorsque le score s'élève en moyenne à 37.01 (ET : 10,26). L'alpha de Cronbach pour cette échelle était de .89 chez les sujets déprimés (Beck, Steer et Brown, 1998). $(\alpha=$ .82 pour notre recherche).

Le versant alexithymique. Le Bermond-Vorst Alexithymia Questionnaire (1994) a été proposé en t0 et t1. Il comprend 40 items regroupés en cinq dimensions théoriques (considérées comme indépendantes) de l'alexithymie : la verbalisation (capacité à décrire ou communiquer ses émotions), la vie fantasmatique (penchant à imaginer, rêverie éveillée), l'identification de ses émotions (capacité à définir ses émotions, à les nommer), l'excitabilité émotionnelle (capacité à être émotionnellement stimulé par un évènement inducteur d'émotions) et l'analyse de ses émotions (capacité à expliquer ses émotions, à les analyser). Les participants avaient à indiquer sur une échelle de Lickert en cinq points, allant de 1 («tout à fait d'accord») à 5 («tout à fait en désaccord ») dans quelle(s) mesure(s) les phrases présentées correspondent à leur manière habituelle de réagir. Plus le score total est élevé, plus la personne est alexithymique. Le questionnaire a été validé auprès de la population hollandaise, anglaise et belge francophone par Bermond et Vorst (1997). L'Alpha de Cronbach pour cet outil varie de .71 à .81 pour la version française. $(\alpha=.70$ pour notre recherche).

La distorsion corporelle. Les sujets ont aussi rempli en t0 et t1 le Body Distorsion Questionnaire créé à l'origine par Fisher (1964) puis réadapté par Sudres et Fourasté en 1984. Cet outil est un indice fiable et valide de la distorsion de l'image corporelle. Il comporte 82 items 
regroupés en sept catégories : «Elargissement », «Rétrécissement », «Perte des frontières », «Blocage des ouvertures », «Peau » (sensation inhabituelle), « Saleté » et « Dépersonnalisation » (perception du corps comme étranger, aliéné ou dévitalisé). Les items sont des questions posées de façon affirmatives. Le sujet coche la case « oui » ou « non ». Le score total se calcule en additionnant les résultats de chacun des facteurs. A partir du moment où ce score est supérieur à 10, nous pouvons parler d'une distorsion corporelle effective (Fisher, 1970). $(\alpha=.72$ pour notre recherche). [32]

Le vécu des ateliers. 2 outils ont été proposés afin d'évaluer le vécu à plusieurs moments : pendant les séances et après l'ensemble des ateliers. D'une part, l'Echelle Clinique de Thérapies Médiatisées (ECTM) a été créée par Sudres en 1993. Elle est composée de 16 items appréhendant le vécu des ateliers autour de 4 thèmes : «définition de l'atelier », «travail psychique », «perception/ attentes du patient » et « processus de changement ». Cet outil donne une vision globale de tout ce que la personne a pu vivre au cours des ateliers de Musicothérapie et favorise une adaptation rigoureuse des activités en fonction d'une population observée. D'autre part, à chaque séance, les sujets ont rempli une échelle construite pour les besoins de l'étude, décrivant l'état de la personne « avant », «pendant » et «après » l'atelier. La personne avait à entourer pour chacun des énoncés un visage parmi les quatre (allant du plus triste au plus joyeux) correspondant le plus à son état et pouvait rajouter des remarques. Cet outil permet de réaliser des graphiques illustrant des mouvements internes qui évolueraient au cours de l'atelier et pouvant échapper à l'observation de l'animateur du groupe (Graphique 1).

La créativité. Une approche qualitative a été choisie pour appréhender ce domaine. Celleci se base sur la production écrite, notamment dans le cadre de la création d'un CD groupal. Un Protocole d'Apperception Thématique Sonore (PATS) a également été construit en se référant 
aux indications de Sorabella [30]. Il s'agit d'un test projectif, s'apparentant à un «TAT » sonore. En fonction des sujets rencontrés, 6 extraits sonores ont été constitués, chacun comportant une séquence de plusieurs sons. Ces extraits, censés proposer par cette succession de sons une histoire, comprennent un contenu latent ainsi qu'un contenu manifeste. Dans ce travail, il n'a été qu'un support de verbalisation, mais il est prévu par la suite de l'utiliser comme outil de mesure de la créativité.

\section{Analyses statistiques}

L'analyse de statistiques descriptives a été utilisée afin de décrire la distribution de chacune des variables en termes de moyennes, de fréquences (Tableau 1) ou d'écart-types en pré- et post-test. Des techniques d'analyses statistiques inférentielles ont ensuite été appliquées. Les conditions d'homogénéité, de normalité n'étant pas respectées et vu la taille de l'échantillon, des tests nonparamétriques de classement ont été choisis pour analyser les variables quantitatives (Wilcoxon) et qualitatives (McNemar). La taille d'effet ( $r$ de Pearson ; $v$ de Cramer) a aussi été calculée. L'ensemble des analyses a été effectué à l'aide du logiciel SPSS.

\section{Résultats}

Le test de Wilcoxon montre que plusieurs scores en post-test (t1) sont significativement différents par rapport à la situation en pré-test (t0). Tout d'abord, ce test indique une augmentation significative entre le $\mathrm{t} 0(\mathrm{Md}=105)$ et le $\mathrm{t} 1(\mathrm{Md}=121)$ au score total de Mindfulness, $\mathrm{Z}=-3.04, p<.002$, et la taille d'effet était relativement grande $: r=-.55$. Il y a eu une augmentation significative entre le t0 $(\mathrm{Md}=22)$ et le $\mathrm{t} 1(\mathrm{Md}=31)$ à propos du score de 
l'Observation, $\mathrm{Z}=-3.29, p<.001$, et la taille d'effet était relativement importante $: r=-.60$. Nous notons une augmentation significative entre le t0 $(\mathrm{Md}=18)$ et le $\mathrm{t} 1(\mathrm{Md}=24)$ au score de Description, $\mathrm{Z}=-3.22, p<.001$, et la taille d'effet était relativement grande : $r=-.58$. Une augmentation significative est aussi à relever entre le t0 $(\mathrm{Md}=19)$ et le $\mathrm{t} 1(\mathrm{Md}=21)$ à propos $\mathrm{du}$ score correspondant à l'Action/agir en pleine conscience, $Z=-2.01, p<.045$, et la taille d'effet semblait moyenne : $r=-.37$. Il y a eu une augmentation significative entre le t0 $(\mathrm{Md}=19)$ et le t1 $(\mathrm{Md}=21)$ de la capacité d'Acceptation, $\mathrm{Z}=-2.69, p<.007$, et la taille d'effet paraissait moyenne : $r=-.49$. A propos du degré de l'état dépressif, il y a eu une diminution significative entre le t0 $(\mathrm{Md}=36)$ et le t1 $(\mathrm{Md}=25), \mathrm{Z}=-3.41, p<.001$, et la taille d'effet était relativement grande $: r=-$ .62. Une diminution significative est aussi constatable entre le t0 $(\mathrm{Md}=125)$ et le t1 $(\mathrm{Md}=110)$ au score total d'Alexithymie, $\mathrm{Z}=-3.32, p<.001$, et la taille d'effet était relativement importante : $r=-.61$. Nous observons une diminution significative entre le t0 $(\mathrm{Md}=29)$ et le $\mathrm{t} 1(\mathrm{Md}=25)$ concernant le score de Verbalisation (des émotions), $\mathrm{Z}=-2.98, p<.003$, et la taille d'effet semblait grande : $r=-.54$. Une diminution significative est à noter entre le t0 $(\mathrm{Md}=30)$ et le $\mathrm{t} 1(\mathrm{Md}=27)$ au score de la Vie Fantasmatique, $\mathrm{Z}=-2.24, p<.025$, et la taille d'effet paraissait moyenne : $r=-$ 41. Le même constat peut être effectué entre le t0 $(\mathrm{Md}=28)$ et le t1 $(\mathrm{Md}=24)$ concernant le score d'Identification des émotions, $\mathrm{Z}=-2.24, p<.025$, avec une taille d'effet semblant moyenne : $r=-.41$. Au niveau corporel, le test indique une diminution significative entre le t0 $(\mathrm{Md}=17)$ et le t1 $(\mathrm{Md}=8)$ au score total de Distorsion, $\mathrm{Z}=-3.41, p<.001$, et la taille d'effet était relativement grande : $r=-.62$. Nous notons une diminution significative entre t0 $(\mathrm{Md}=3)$ et $\mathrm{t} 1(\mathrm{Md}=0)$ concernant le score de Perte des Frontières, $\mathrm{Z}=-2.93, p<.003$, et la taille d'effet était relativement grande : $r=-.053$. Il y a eu aussi une diminution significative entre le t0 $(\mathrm{Md}=5)$ et le $\mathrm{t} 1(\mathrm{Md}=2)$ au score de Blocage des ouvertures, $\mathrm{Z}=-3.13, p<.002$, et la taille d'effet était relativement 
grande $: r=-.57$. Enfin, nous remarquons une diminution significative entre le t0 $(\mathrm{Md}=7)$ et le $\mathrm{t} 1$ $(\mathrm{Md}=3)$ en termes de Dépersonnalisation corporelle, $\mathrm{Z}=-3.07, p<.002$, et la taille d'effet était relativement grande : $r=-.56$.

Le test de McNemar montre une apparition significative de deux défenses matures en situation de post-test. Celui-ci indique que la présence de la Sublimation a augmenté significativement au cours de la période d'ateliers entre t0 et $\mathrm{t} 1(p<.01)$. Les patients semblaient présenter d'avantage de sublimation en post-test $(86.70 \%)$ qu'en pré-test $(33.33 \%)$. Nous constatons une taille d'effet relativement importante : $v=.76$. De plus, la présence de la Répression a aussi augmenté significativement durant la période d'ateliers entre t0 et $\mathrm{t} 1(p<.001)$. Les patients semblaient présenter d'avantage de répression en post-test (93.30\%) qu'en pré-test (13.30\%). La taille d'effet s'avérait également relativement importante $: v=.93$

\section{Discussion}

En comparant les situations pré- et post-test, nos résultats mettent en exergue des améliorations concernant l'aptitude à focaliser sa conscience dans le présent (Mindfulness global, Observation, Description, Action/ agir en pleine conscience, Acceptation) ainsi qu'une atténuation de la sévérité de la dépression. Des progrès sont d'ailleurs cités par les participants (Tableau 2). En effet, selon eux, l'atelier représente un soutien dans le processus de guérison [25] en procurant de l'apaisement, du repos ou encore en permettant de ressentir et apprécier l'instant présent. Réciproquement, ceci pourrait consolider une tendance à la rêverie plus importante (cf. scores Vie fantasmatique). Les sujets ont aussi perçu un sentiment d' «ouverture à la nouveauté et à l'extérieur» qui semblerait en lien avec une sensation d'Ouverture corporelle (cf. BDQ). D'autres améliorations émotionnelles sont relevables (Alexithymie globale, Verbalisation, Iden- 
tification des émotions). Parmi les ressentis les plus saillants (Tableau 2), nous retrouvons le renforcement du lien social, favorisé par le partage musical (notamment le chant). Le lâcher prise, la détente groupale, sont vécus par certains comme une «sortie d'eux-mêmes » et paraissent accroître l'échange verbal. L'instrument de musique se situant entre un vécu corporel et une parole [30], nous pouvons supposer que les progrès concernant la Distorsion globale, la Perte des frontières, et la Dépersonnalisation (dévitalisation corporelle) aient pu favoriser une meilleure prise de conscience d'un monde physiologique émotionnel interne (Identification), et donc d'un passage à la Verbalisation. Ce sont aussi ces échanges musicaux, puis verbaux qui ont conduit à la création groupale. Ainsi, si nous tenons compte du renfort du lien (malgré un groupe « hétérogène »), de l'illusion groupale évoqués (Tableau 2) ou de la création du $\mathrm{CD}$, il semblerait logique que la Sublimation soit une défense dont la présence s'est amplifiée. De plus, pour Bergeret [30], ce qui est sublimé en art n'est pas l'objet mais l'activité artistique en elle-même: peu importe la valeur du résultat, la sublimation ne produit pas que du « sublime », la créativité peut être entendue comme un produit de cette sublimation. En outre, la Répression, oubli réversible et fonctionnel, mise à l'écart des éléments perturbants dans le préconscient, restant ainsi accessibles [12], est également d'avantage observée après l'ensemble des séances. Bien qu'il faille prendre garde à ce que celle-ci ne soit pas excessive à l'avenir, ces résultats sont aussi en lien avec les propos tenus par certains patients lorsqu'ils abordent les améliorations perçues (Tableau 2). En ce sens, en ajout à une sensation de bien-être, de progrès technique (surmonter une voix monocorde), d'une meilleure attention aux sons entendus, du passage d'une écoute passive à une écoute active, ils discernent la possibilité de «laisser tomber les soucis » au cours de l'atelier. Or, ce phénomène serait-il lié à une simple fonction contenante du groupe (Tableau 2) ou à un réel effet musicothérapeutique ? Il importera de s'intéresser d'avantage à quelle place prend par exemple 
la musique dans la vie de la personne : serait-elle susceptible de passer avant le groupe ?

Concernant les fonctions attendues de l'animateur, le praticien devrait avoir un rôle d'interface, de réassurance, de contenance et de régulateur thymique (Tableau 2), obligeant ainsi le Musicothérapeute à adapter constamment une humeur opposée à la leur. De plus, trois éléments primordiaux sont à aborder : la fin, la culture et la représentation de la normalité. Le rôle de l'annonce de la fin a été crucial puisqu'elle a enclenché le processus créatif en générant du manque chez les sujets (et donc du désir). En effet, bien qu'elle fasse peur, la fin a engendré ce processus qui a permis aux sujets de se restructurer, voire d'exister ou se projeter au-delà de cette finalité. Au niveau culturel, avec la présence de nationalités différentes (Tableau 1) nous notons la résonnance d'une troisième culture, ainsi que d'un troisième lieu, qui, par le biais de la musique, permet de donner une place à chacun. Enfin, la rencontre entre un Moi triste et un autre triste était impossible tant l'état de l'autre était parfois insupportable (33\%) : serait-ce dû à une norme trop envahissante (cf. perte du droit d'être triste [8,23]) malgré une compréhension mutuelle ? Ou estce que l'autre représenterait un miroir intolérable?

Le vécu par séance montre que les états positifs étaient majoritairement perçus pendant les ateliers et après (mais dans de plus faibles proportions). Les données recueillies avec l'ECTM nous permettent de préciser : après un amorçage nécessitant un effort (40\%), la séance était ressentie comme étant agréable (100\%), pouvant par exemple produire du bien-être (27\%) ou une ouverture aux autres (60\%). Or, il sera important d'interroger le fait que $26 \%$ des sujets expriment une certaine résistance au changement («triste, je le suis toujours »). En ajout au followup, il serait pertinent de créer dans une recherche à venir, des séances post-production du $\mathrm{CD}$ autour de la représentation suivante : «est- ce que je peux changer ?». 
Si nous regardons le bien-être perçu (Tableau 2), les sujets évoquent la possibilité de s'évader des contraintes temporelles de l'environnement hospitalier: avoir un autre rythme, «gérer le temps » en «reprenant confiance en la vie ». Dans cette temporalité particulière, en plus d'une régularité fondamentale (unité de lieu et de durée des ateliers), une ritualisation interne s'est progressivement établie. Celle-ci a pu ainsi apporter une certaine rythmicité, une pulsation, dans un cadre où le quotidien hospitalier est donc difficile à supporter. La « ritournelle » est la suivante :

Mise en voix individuelle $\rightarrow$ Mise en voix collective $\rightarrow$ Mise en corps $\rightarrow$ Création

En ce sens, après un amorçage nécessitant un effort notamment en matinée (Tableau 2) et en début de séance (Figure 1), seules l'écoute (temps faible de l'atelier) ou l'utilisation de la voix étaient faisables. Tel un minimum vital de résilience s'apparentant au « spleen » Baudelairien, la mise en vibration intérieure et impérativement différente de celle des autres (sous-peine d'angoisse) était acceptable. Il s'agissait d'une forme de mise en sécurité individuelle dont la consigne aurait pu être «C'est vous qui vous mettez en activité d'existence ». Ensuite, la vibration vocale extérieure s'apparentait à une mise en sécurité collective, permettant une existence intersubjective et/ ou culturelle. Enfin, le jeu instrumental en groupe (mouvement) constituait la dernière passerelle avant la création groupale et/ ou individuelle (cf. CD).

Cependant, l'étude s'effectuant en milieu naturel et étant en cours, la petite taille de l'échantillon et la monocentricité de la recherche ne permettent pas une réelle représentativité de la population rencontrée. Ainsi, concernant le déroulement de la recherche à venir, l'échantillon se constituera sur trois ans (objectif : 40 sujets par groupes) dans le cadre d'une étude multicentrique. De plus, nous ne pouvons pas affirmer que ce sont précisément ces ateliers qui ont eu les 
impacts décrits. Il importera donc de créer deux groupes supplémentaires : un groupe contrôle (prise en charge habituelle) et un groupe d'échange autour du thème de la musique (en ajout à la prise en charge habituelle). Cette étude n'est pas non plus préservée des biais de désirabilité sociale, des effets nocebo ou placebo. Il est néanmoins prévu qu'un follow-up soit réalisé systématiquement afin d'évaluer les effets et l'efficience à long terme de cette approche. Il sera également intéressant d'observer quelles aires cérébrales sont atteintes par la sismothérapie afin de rechercher celles que la Musicothérapie peut stimuler. Enfin, des recommandations de bonnes pratiques d'un programme structuré et multidimensionnel à médiation musicale de la problématique dépressive résistante seront suggérées en 2018. 


\section{Références}

[1] Abbass, A. (2006). Intensive Short-Term Dynamic Psychotherapy of treatment-resistant depression: a pilot study. Depression And Anxiety, 23(7), 449-452

[2] Baas, M., Nijstad, B. A., Boot, N. C., \& De Dreu, C. W. (2016). Mad Genius Revisited: Vulnerability to Psychopathology, Biobehavioral Approach-Avoidance, and Creativity. Psychological Bulletin, doi: 10.1037/bul0000049

[3] Berlim, M. G. (2007). Definition, Assessment, and Staging of Treatment-Resistant Refractory Major Depression: A Review of Current Concepts and Methods. Canadian Journal Of Psychiatry, 52(1), 46-54

[4] Bermond B., Vorst H-C. (1994). Alexithymia a conjunctive model of traits. Manuscrit inédit

[5] Bermond B., Vorst H-C., Luminet, O., Rimé, B., Zech, E. et Wagner H. (1998). Validity and reliability of Bermond-Vorst alexithymia questionnaire. Texte soumis

[6] Beuzen, J-N. (2015). La musique, entre génie créateur et vertu thérapeutique, Paris, Odile Jacob

[7] Blashill, A. J., \& Wilhelm, S. (2014). Body image distortions, weight, and depression in adolescent boys: Longitudinal trajectories into adulthood. Psychology Of Men \& Masculinity, 15(4), 445-451. doi:10.1037/a0034618

[8] Bouvard, M. (2015). Les troubles psychiatriques résistants, PUG (Presses Universitaires de Grenoble)

[9] Bréjard V., Bonnet A ., Pedinielli J-L (2008), Emotion regulation, depression and risk-taking behaviour: alexithymia as a moderating factor, Annales Médico-psychologiques, revue psychiatrique, Elsevier, 260-268 
[10] Campos, R.C., Besser, A., \& Blatt, S. J. (2011). The relationships between defenses and experiences of depression: An exploratory study. Psychoanalytic Psychology, 28(2), 196-208. doi:10.1037/a0022420

[11] Chabrol H. (2005). Les mécanismes de défense., Recherche en soins infirmiers 3 ( $\left.\mathrm{N}^{\circ} 82\right)$, p. 31-42. doi : 10.3917/rsi.082.0031

[12] Chabrol, H., Callahan, S. (2013). Mécanismes de défense et coping. $2^{\text {ème }}$ édition, Paris, Dunod

[13] Coleman, D., Cole, D., \& Wuest, L. (2010). Cognitive and psychodynamic mechanisms of change in treated and untreated depression. Journal of clinical psychology, 66(3), 215-228

[14] Eckhardt, K. J., \& Dinsmore, J. A. (2012). Mindful Music Listening as a Potential Treatment for Depression. Journal Of Creativity In Mental Health, 7(2), 175-186. doi:10.1080/15401383.2012.685020

[15] Foran, H. D. (2013). The Role of Relationships in Understanding the AlexithymiaDepression Link. European Journal Of Personality, 27(5), 470-480

[16] Forestier, R (2011). Tout savoir sur la Musicothérapie, Paris, Favre

[17] Gérard, A. (2010). Dépression, la maladie du siècle, Paris, Albin Michel

[18] Heeren, A., Douilliez, C., Peschard, V., Debrauwere, L., \& Philippot, P. (2011). Crosscultural consistency of the Five Facets Mindfulness Questionnaire: Adaptation and validation in a French sample.Revue Européenne de Psychologie Appliquée, 61, 147-151

[19] Jackson, J. C. (2001). Exploring the Relationship between Schizophrenia, Affective Illness, and Creativity. Available from: ERIC, EBSCOhost

[20] Johnson, S., Murray, G., Hou, S., Staudenmaier, P., Freeman, M., Michalak, E. (2015), Creativity is linked to ambition across the bipolar spectrum, Journal of affective disorder, Volume 178, Pages 160-164. Doi: http://dx.doi.org/10.1016/j.jad.2015.02.021 
[21] Kattenstroth, M. (2009) The relation between body-self-unity and mindfulness : a comparison of currently depressed patients and exercisers. University of Twente Student Theses

[22] Lecourt, E. (2014). La Musicothérapie. Paris, Eyrolles

[23] Le Blanc, G. (2004), Les maladies de l'homme normal, Paris, Editions du passant

[24] Le Breton, D. (2015), Disparaître de soi, Paris, Métailié

[25] Lee, J., \& Thyer, B. (2013). Does Music Therapy Improve Mental Health in Adults? A Review (English). Journal Of Human Behavior In The Social Environment, 23(5-8), 591-603

[26] Mirabel-Sarron, C., Leygnac-Solignac I. (2015). Les troubles bipolaires, de la cyclothymie au syndrome maniaco-dépressif, Paris, Dunod

[27] Petchkovsky, L., Robertson-Gillam, K., Kropotov, J., \& Petchkovsky, M. (2013). Using QEEG parameters (asymmetry, coherence, and P3a novelty response) to track improvement in depression after choir therapy. Advances In Mental Health, 11(3), 257-267.

doi:10.5172/jamh.2013.11.3.257

[28] Rizvi, S. H. (2014). Treatment-Resistant Depression in Primary Care Across Canada. Canadian Journal Of Psychiatry, 59(7), 349-357

[29] Siedliecki, S. L., \& Good, M. (2006). Effect of music on power, pain, depression and disability. Journal Of Advanced Nursing, 54(5), 553-562. doi:10.1111/j.1365-2648.2006.03860.x

[30] Sorabella, F. (2015, 13 février). L'approche Créative dans le dispositif musicothérapique, de l'utilisation des nouvelles technologies. [Vidéo en ligne]. Repéré à https://www.youtube.com/watch?v=sK4TOZAcfrk 
[31] Speranza, M., Corcos, M., Loas, G., Stéphan, P., Guilbaud, O., Perez-Diaz, F. et al.

Depressive personality dimensions and alexithymia in eating disorders. Psychiatry Res. 2005 [cité le 15 juin 2005] 15;135(2):153-63

[32] Sudres, J-L., Moron, P., Roux, G. (2004). Créativité et art-thérapie en psychiatrie. Paris, Broché 
Tableau 1. Description de l'échantillon $(\mathrm{N}=15)$

\begin{tabular}{lll}
\hline Variables socio-biographiques & n & \% \\
\hline Parentalité & 7 & 46.67 \\
sans enfant & 8 & 53.33 \\
$\quad$ un enfant ou plus & & \\
Mode de vie & 7 & 46.67 \\
$\quad$ célibataire/ séparé/ divorcé/ veuf & 6 & 40.00 \\
$\quad$ en couple/ pacsé/ marié & 2 & 13.33 \\
$\quad$ hébergé chez les parents/ établissement de soin & 2 & 46.67 \\
Situation professionnelle & & 13.33 \\
$\quad$ sans emploi, en invalidité, étudiant & 7 & 40.00
\end{tabular}

\section{Origine culturelle}

\begin{tabular}{lll} 
française & 12 & 80.00 \\
pas française & 3 & 20.00 \\
\hline Variables psychologiques & $\mathbf{n}$ & $\mathbf{\%}$ \\
\hline $\begin{array}{l}\text { Type de prise en charge } \\
\text { hospitalisation }\end{array}$ & 12 & 80.00 \\
ambulatoire & 3 & 20.00 \\
hospitalisation avec sismothérapie & 9 & 60.00 \\
Diagnostic & & 26.67 \\
$\quad \begin{array}{l}\text { dépression résistante unipolaire } \\
\text { dépression résistante bipolaire } \\
\text { mélancolie }\end{array}$ & 4 & 33.33 \\
trouble schizo-affectif & 5 & 33.33 \\
& 5 & 6.67 \\
\hline Variables en lien avec le rapport à la musique & 1 & $\mathbf{\%}$ \\
\hline Pratique de la musique durant l'enfance et/ ou l'adolescence & & 40.00 \\
\hline Pratique de la musique actuellement & $\mathbf{n}$ & 6.67 \\
\hline & 6 & 1 \\
\hline
\end{tabular}


Tableau 2. Synopsis des résultats de l'Echelle Clinique des Thérapies Médiatisées

\section{Objectifs de l'atelier}

\begin{tabular}{|c|c|}
\hline $\begin{array}{l}\text { Soutien dans le processus de guérison } \\
\qquad 60.00 \%\end{array}$ & $\begin{array}{ll}\text { - } & \text { Repos, apaisement } \\
\text { - } & \text { Ouverture à la nouveauté, à l'extérieur, } \\
\text { - } & \text { Etre dans le présent, ressentir et apprécier l'instant }\end{array}$ \\
\hline Bien-être perçu & $\begin{array}{ll}\text { - } & \text { Détente } \\
\text { - } & \text { Evasion du quotidien hospitalier } \\
\text { - } & \text { Passer un moment agréable }\end{array}$ \\
\hline Être- ensemble & $\begin{array}{ll}\text { - } & \text { Etre en lien par le biais du chant groupal } \\
\text { - } & \text { Se détendre ensemble } \\
\text { - } & \text { Découvrir les goûts de chacun } \\
\text { - } & \text { L'animateur a pour fonction de transmettre musicalement au } \\
& \text { maximum, ou encore de faire aimer la musique }\end{array}$ \\
\hline \multicolumn{2}{|c|}{ Le travail psychique } \\
\hline
\end{tabular}

- Création groupale conduisant au plaisir d'être ensemble (60.00\%)

- Ecoute plus aisée, notamment lorsque l'atelier se déroule le matin (40.00\%)

- Chant : tremplin principal permettant d'amener chacun à se surprendre soi-même (46.67 \%), favorisant l'échange musical puis une tolérance mutuelle (30.00\%)

- La séance est perçue comme agréable (100\%) malgré un amorçage (matinal) nécessitant souvent un effort $(40.00 \%)$

- Difficulté de supporter le «bruit» de l'autre (20.00\%), aggravant une fatigue constante

\section{La perception/ Les attentes du patient}

\begin{tabular}{|l|cl|}
\hline $\begin{array}{l}\text { Amélioration des capacités, percep- } \\
\text { tions, sensations }\end{array}$ & - & Progrès technique instrumental mentionné \\
\multicolumn{1}{|c|}{$73.33 \%$} & - & Passage d'une écoute « passive » à une écoute active \\
& - & Meilleure attention aux sons entendus \\
& - & Amélioration des aptitudes à contenir ses émotions \\
\hline Fonctions attendues de l'animateur & - & Transmettre, intégrer et écouter la demande de chacun dans le \\
\hline
\end{tabular}




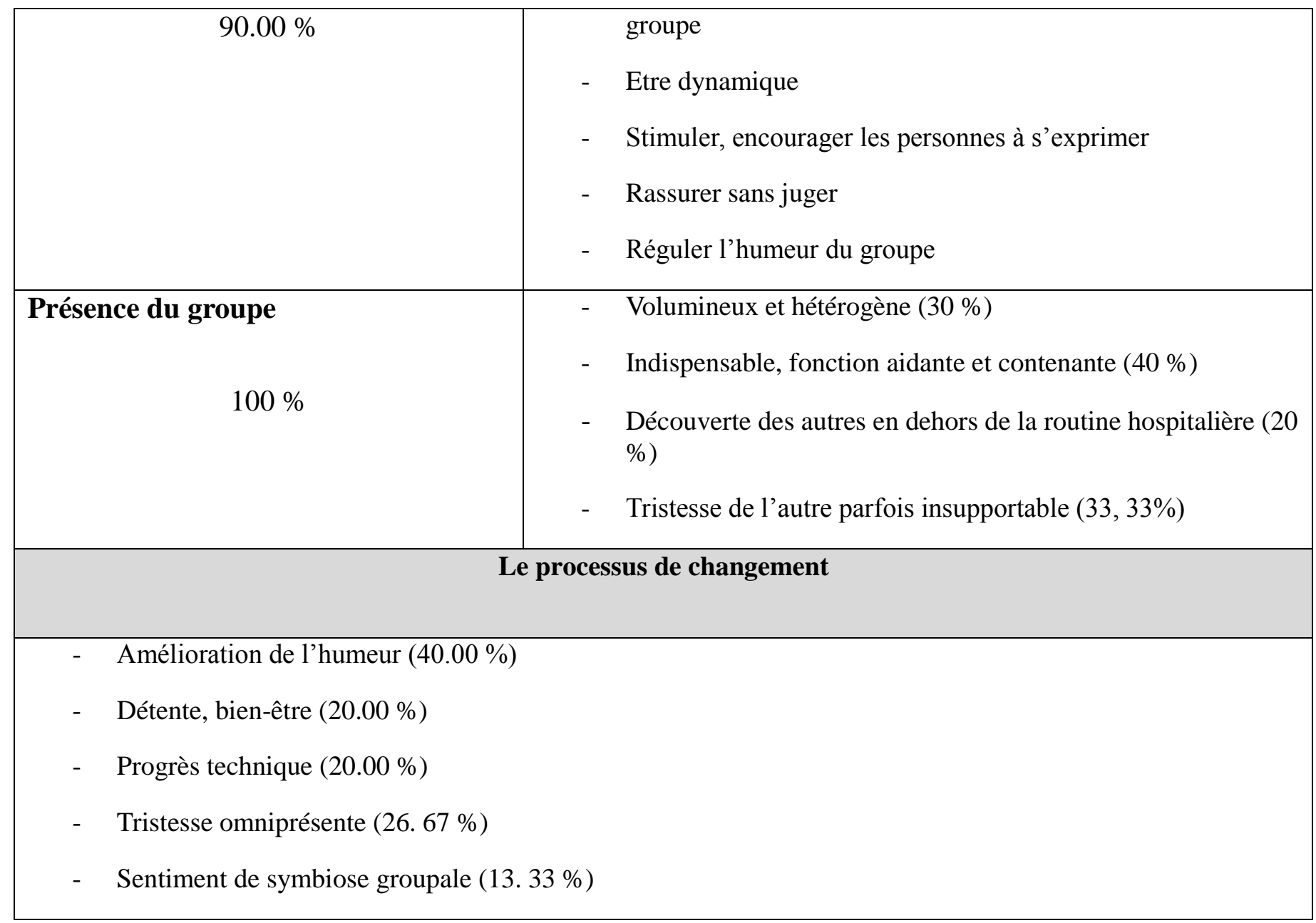




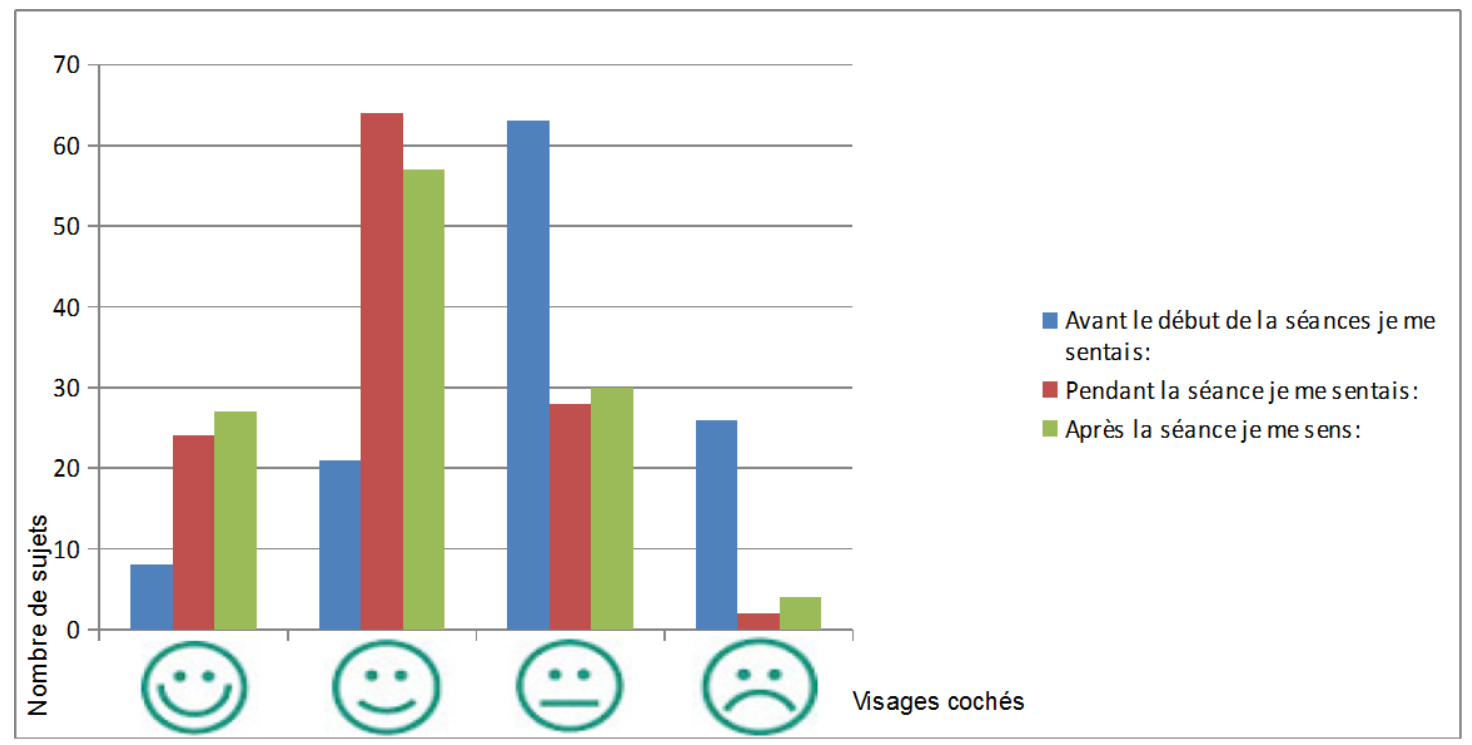

Figure 1. Le vécu par séance 